\title{
Metabolic network analysis and experimental study of lipid production in Rhodosporidium toruloides grown on single and mixed substrates
}

\author{
Rajesh Reddy Bommareddy ${ }^{\dagger}$, Wael Sabra $^{\dagger}$, Garima Maheshwari and An-Ping Zeng ${ }^{*}$
}

\begin{abstract}
Background: Microbial lipids (triacylglycerols, TAG) have received large attention for a sustainable production of oleochemicals and biofuels. Rhodosporidium toruloides can accumulate lipids up to $70 \%$ of its cell mass under certain conditions. However, our understanding of lipid production in this yeast is still much limited, especially for growth with mixed substrates at the level of metabolic network. In this work, the potentials of several important carbon sources for TAG production in R.toruloides are first comparatively studied in silico by means of elementary mode analysis followed by experimental validation.
\end{abstract}

Results: A simplified metabolic network of R.toruloides was reconstructed based on a combination of genome and proteome annotations. Optimal metabolic space was studied using elementary mode analysis for growth on glycerol, glucose, xylose and arabinose or in mixtures. The in silico model predictions of growth and lipid production are in agreement with experimental results. Both the in silico and experimental studies revealed that glycerol is an attractive substrate for lipid synthesis in $R$. toruloides either alone or in blend with sugars. A lipid yield as high as 0.53 (C-mol TAG/C-mol) has been experimentally obtained for growth on glycerol, compared to a theoretical maximum of 0.63 (C-mol TAG/C-mol). The lipid yield on glucose is much lower (0.29 (experimental) vs. 0.58 (predicted) C-mol TAG/C-mol). The blend of glucose with glycerol decreased the lipid yield on substrate but can significantly increase the overall volumetric productivity. Experimental studies revealed catabolite repression of glycerol by the presence of glucose for the first time. Significant influence of oxygen concentration on the yield and composition of lipids were observed which have not been quantitatively studied before.

Conclusions: This study provides for the first time a simplified metabolic model of R.toruloides and its detailed in silico analysis for growth on different carbon sources for their potential of TAG synthesis. Experimental studies revealed the phenomenon of catabolite repression of glycerol by glucose and the importance of oxygen supply on the yield and composition of lipids. More systematic studies are needed to understand the mechanisms which should help to further optimize the lipid production in this strain of industrial interest.

Keywords: Metabolic network analysis, Elementary mode analysis, Lipids, Glycerol, Biomass, Oleaginous yeast

\section{Background}

During the past years fuel production from biomass has gained enormous interest due to the escalating cost and scarcity of fossil fuels. Biodiesel industry has become important and the use of plant oils as raw materials is not ecologically sustainable because of the relatively high production costs and their competition with global

\footnotetext{
* Correspondence: aze@tuhh.de

${ }^{\dagger}$ Equal contributors

Institute of Bioprocess and Biosystems Engineering, Hamburg University of Technology, Denickestrasse 15, D-21073 Hamburg, Germany
}

supply of food. Despite the expected high production capacity of biodiesel the anticipated target has increased at a slower rate [1], mainly due to its relatively high production cost. However, utilization of glycerol, a coproduct from biodiesel production, is one of the promising options for lowering its production cost $[2,3]$. In fact, biodiesel production generates about $10 \%(\mathrm{w} / \mathrm{w})$ glycerol as the main byproduct. Therefore, many researchers have worked on valorization of glycerol and successfully produced value added chemicals which are industrially significant [4-8]. 
On the other hand, microbial lipids are being explored as raw materials for the production of biodiesel and functional oils with comparable properties of those produced from plant oils [9-11]. Moreover, microbial substitutes of industrially important products like cocoa butter from oils produced by microorganism has been previously reported [12]. An important advantage offered by the application of the oleaginous microorganisms is their ability to produce lipids from cheep substrates. Lignocellulosic biomass is considered as the only foreseeable, feasible and sustainable resource for renewable fuel, and large efforts have been implemented worldwide to replace the first generation of fuels based on high-value sugars and oils with $2^{\text {nd }}$ generation biofuels based on cheaper and more abundant lignocellulosic biomass. In fact, several oleaginous microorganisms were reported for efficient lipid production using industrial wastes and biomass hydrolysate [2,13-18]. Since glycerol is the backbone of microbial lipids, it is of interest to examine if glycerol, especially glycerol from biodiesel production, can be effectively used together with biomass hydrolysate. Few studies have been reported on the use of mixed substrates for the synthesis of microbial lipids.

Several yeast strains are known for lipids production, which include Cryptococcus albidus, Lipomyces lipofera, Lipomyces starkeyi, Rhodosporidium toruloides, Rhodotorula glutinis, Trichosporon pullulan, and Yarrowia lipolytica [19]. Ageitos et al. [19] compared the different yeast strains in terms of productivity and yield, of which $R$. toruloides and $R$. glutinis were shown to be the most promising. Y. lipolytica was also proven to be a potential lipid producer, especially after improvement with genetic tools.

The red yeast Rhodosporidium toruloides has a high capability for growth and lipid synthesis on a range of carbon sources from glucose, fructose and xylose to glycerol [20-22]. Lipids can accumulate to a concentration of $60 \%(w / w)$ in cell mass of $R$. toruloides. Its ability to simultaneously assimilate sugars, especially glucose and glycerol has not been studied in detail. Studies on a closely related species $R$. glutinis showed that glucose and glycerol can be utilized simultaneously with preference toward glycerol which is similar to $Y$. lipolytica [23].

With substrate cost and availability being continually changing, the utilization of multiple feed stocks is crucial for process viability. Therefore the aim of this study was to analyze the metabolic network and fluxes of $R$. toruloides grown on different substrates and substrate mixtures, using detailed biochemical knowledge on genome scale. Elementary mode analysis was used to elucidate the optimal pathway and the various fluxes for lipid accumulation from different substrates. Validation experiments were then performed and the kinetics of growth, lipid production and substrate uptake were evaluated. Experiments with biomass hydrolysate as pure substrates or with glycerol were also compared. Since lipid production is a process requiring intensive reducing power and energy, the control of oxygen supply on lipid accumulation and composition is important and studied in a wellcontrolled bioreactor system. An understanding of these metabolic processes can open doors for metabolic engineering of this yeast and for process optimization.

\section{Results and discussion}

In silico analysis of triacylglycerol production on different substrates

The large quantity of information featured in public databases, like details about genomes, pathways and proteins were first used for the reconstruction of the metabolic network and the in silico modeling of $R$. toruloides. With the genome annotation and other metabolic capacities in available literature and experiments, a draft metabolic network model was validated and curated manually until a high-quality metabolic network model of $R$. toruloides was constructed (see Methods section and Additional file 1: Table S1 for details). On that basis, the influence of different substrates on lipid production was first investigated. Elementary flux modes were estimated for the defined metabolic network of $R$. toruloides grown on four different substrates (glucose, glycerol, xylose and arabinose). The numbers of elementary modes obtained from the metabolic network for each substrate are shown in Table 1. Maximum theoretical yields of TAG were found to be in the modes where there is no cell mass production. This can be meaningful as lipid production is induced by nitrogen limitation where growth limitation occurs. Among the substrates, the glycerol utilizing network yielded more EMs and a higher theoretical maximum yield of TAG whereas among the sugars glucose showed the highest yield. Comparison of the solution spaces spanned by elementary modes on these substrates is represented in Additional file 1: Figure S1.

With glucose as the sole carbon and energy source, the computed optimum flux distribution is shown in Figure 1. The most common product found in $94 \%$ of the modes is cell mass whereas TAG production is found in $25 \%$ of the modes. Maximum theoretical yield of the TAG is found to be $0.07 \mathrm{~mol} . \mathrm{mol}^{-1}$ on the basis of an assumed TAG molecule size of 51 carbons ( $3 \mathrm{X} \mathrm{C}_{16}$ palmitic acid + glycerol 3-phosphate). Taking the molecular weight of the assumed TAG $\left(\mathrm{C}_{51} \mathrm{H}_{92} \mathrm{O}_{6}=801.27 \mathrm{~g} \cdot \mathrm{mol}^{-1}\right)$ into account the resulting maximum yield is $0.30 \mathrm{~g} . \mathrm{g}^{-1} \mathrm{glu}-$ cose. For the production of one mol of TAG, 42 moles of NADPH and 21 moles of ATP are required. From the flux distribution map (Figure 1), 63\% of the required NADPH is mainly supplied by the pentose phosphate pathway (PP) 
Table 1 Elementary modes (EMs) of the metabolic network of Rhodosporidium toruloides for growth on different substrates with corresponding maximum TAG and cell mass yields

\begin{tabular}{|c|c|c|c|c|}
\hline $\begin{array}{l}\text { Carbon } \\
\text { source }\end{array}$ & EMs & $\begin{array}{l}\text { Maximum } \\
\text { yield of TAG } \\
\text { [g.g } .^{-1} \text { substrate] }\end{array}$ & $\begin{array}{l}\text { Maximum } \\
\text { yield of TAG } \\
{[\text { C-mol.C-mol }} \\
\text { substrate] }\end{array}$ & $\begin{array}{l}\text { Maximum cell } \\
\text { mass yield } \\
\text { [C-mol.C-mol } \\
\text { substrate] }\end{array}$ \\
\hline Glucose & 14,164 & 0.30 & 0.58 & 0.67 \\
\hline Glycerol & 33,970 & 0.32 & 0.63 & 0.73 \\
\hline Xylose & 9,476 & 0.29 & 0.55 & 0.66 \\
\hline Arabinose & 15,231 & 0.27 & 0.53 & 0.63 \\
\hline
\end{tabular}

and partially by the cytosolic malic enzyme (values normalized to glucose uptake rate as shown in Figure 1). This high flux through the PP pathway might lead to carbon loss in form of $\mathrm{CO}_{2}$, but also for a better precursor availability and growth. In fact, $37.2 \mathrm{~mol} \mathrm{CO}_{2}$ would be released for the synthesis of one mol TAG. Neither the malic enzyme in the cytosol nor the PP pathway alone can compensate for the required NADPH. The flux through the malic enzyme depends on the cytosolic NADH availability as the malic enzyme functions together with the pyruvate-oxaloacetatemalate cycle (POM cycle). About 10\% ATP is generated by

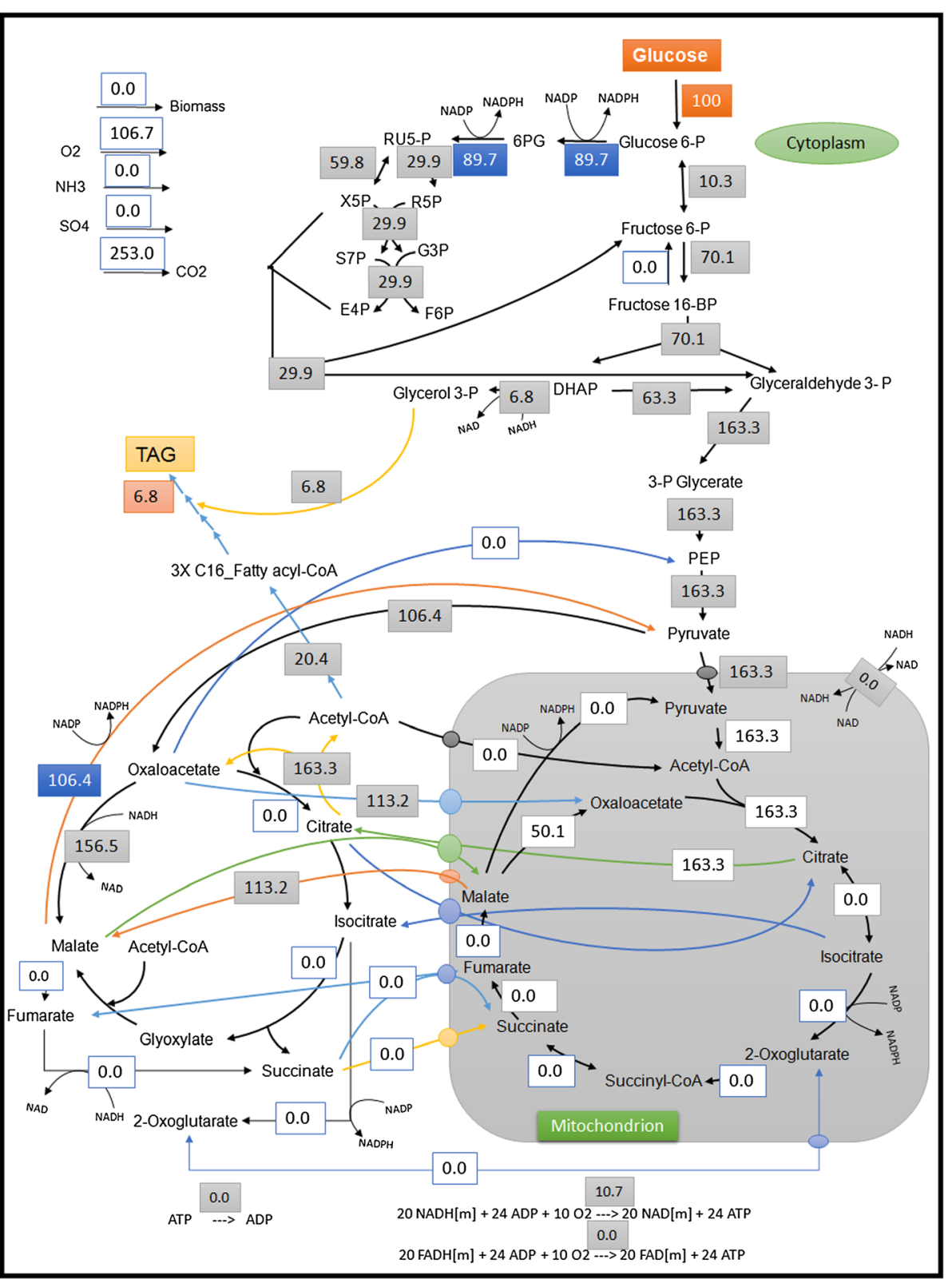

Figure 1 Optimal flux distribution on glucose. All values are relative molar fluxes $\left(\mathrm{mmol} \cdot \mathrm{g}^{-1} \cdot \mathrm{h}^{-1}\right)$ normalized to the glucose uptake rate. 
the respiratory chain reaction using the mitochondrial NADH.

On the other hand, with glycerol as the sole substrate (Figure 2), the NADPH demand is mainly met by the cytosolic malic enzyme whereas there is a reduced PP pathway flux and hence reduced $\mathrm{CO}_{2}$ formation. Glycerol is channeled into glycolysis via the dihydroxyacetone to glyceraldehydes 3-phosphate. Most of the fluxes pass through the lower part of the glycolysis into the pyruvate dehydrogenase complex. Two more optimal modes (data not shown) were identified where similar maximum TAG yields were observed with a succinate to fumarate interconversion in the mitochondrion and cytoplasm. The required ATP is also generated by both the respiratory chain reactions in the mitochondrion.

Similarly, the optimal flux distributions with xylose and arabinose as substrates are shown in Additional file 1: Figures S2 and S3, respectively in the Supplement. Higher numbers of EMs were observed on arabinose compared to xylose. About $96 \%$ of the modes produced cell mass and 23\% of the modes showed TAG production on both the pentoses. A very high PP pathway fluxes is observed on both pentoses, obviously due to the requirement of NADPH for uptake of these substrates [24]. Xylose showed slightly higher theoretical yields of TAG than arabinose. Most of the fluxes are

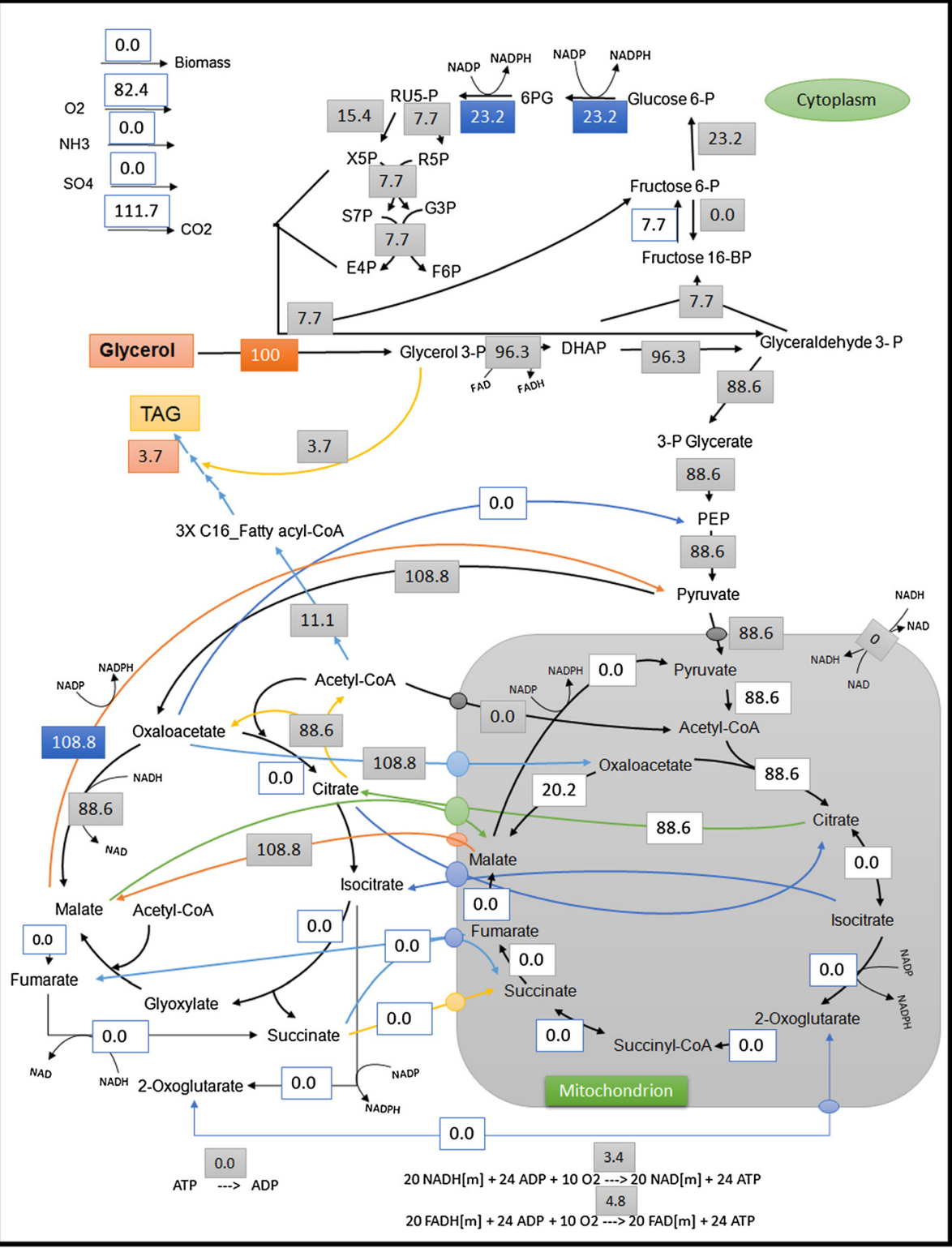

Figure 2 Optimal flux distribution on glycerol. All values are relative molar fluxes $\left(\mathrm{mmol} . \mathrm{g}^{-1} \cdot \mathrm{h}^{-1}\right)$ normalized to the glycerol uptake rate. 
channeled through the gluconeogenic glucose 6phosphate isomerase reaction into the PP pathway on both the pentoses. Similar channeling of fluxes is observed towards the pyruvate dehydrogenase complex on both the substrates. Due to the high demand for $\mathrm{NADPH}$ on arabinose, the oxaloacetate transporter flux into the mitochondrion should be inactive whereas on all the other substrates it should be active for maximal TAG synthesis. This accumulation of oxaloacetate may drive a high flux through the malate dehydrogenase $(\mathrm{MDH})$ in the cytosol towards malate which in turn also increases the flux through the cytolsolic malic enzyme on arabinose than on xylose.
Using the same method, optimal flux distributions and EFM analysis with the proposed metabolic network of $R$. toruloides were performed using blend of substrates. The maximum TAG yield obtained is $0.58 \mathrm{C}_{\mathrm{mol}} \cdot \mathrm{C}_{\mathrm{mol}}{ }^{-1}$ substrate with a blend of glucose and glycerol (Figure 3), compared to $0.55 \mathrm{C}_{\mathrm{mol}} \cdot \mathrm{C}_{\mathrm{mol}}{ }^{-1}$ substrate with a blend of xylose and glycerol (Additional file 1: Figure S4). The represented modes are the best modes consuming both the substrates. Comparing the yields from these analyses, similar maximum TAG yields are observed compared to the yields where single sugars are used (Table 1). Adding glycerol uptake did not show any effect on maximum TAG yields in these in silico analyses. $\mathrm{CO}_{2}$ produced per

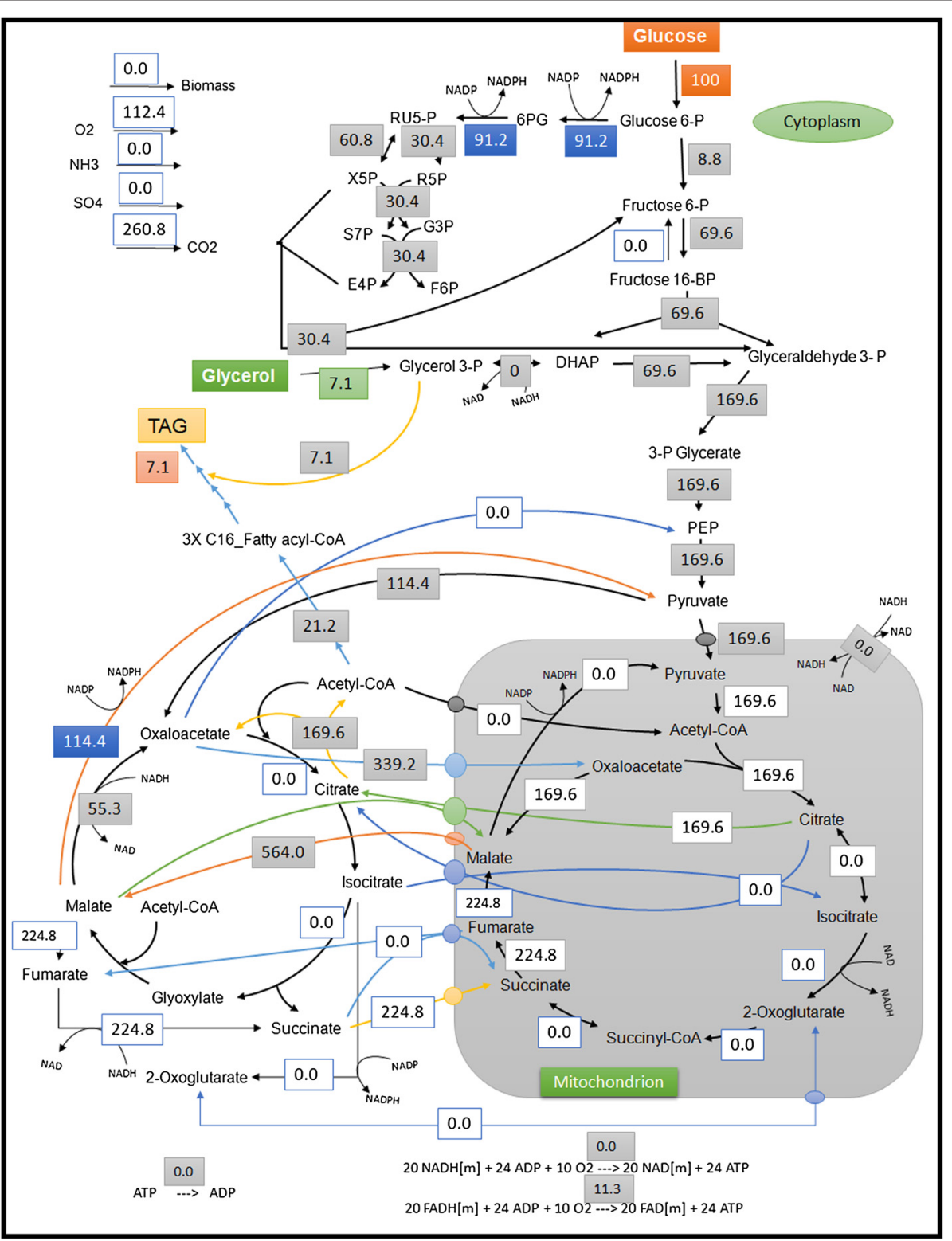

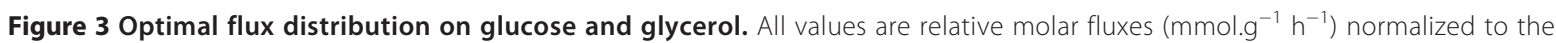
glucose uptake rate. 
mole TAG on different carbon sources are compared from the in silico analyses, arabinose as a carbon source has the highest production of $\mathrm{CO}_{2}$ per TAG molecule (45.6 $\mathrm{mol} / \mathrm{mol}$ TAG), whereas glycerol as a sole carbon source has the lowest $\mathrm{CO}_{2}$ production $(30.7 \mathrm{~mol} / \mathrm{mol}$ TAG), indicating that glycerol is a desirable substrate in terms of efficiency of carbon utilization. $\mathrm{CO}_{2}$ production is proportional to the flux entering into the PP pathway as clearly observed from the in silico predictions.

\section{Controlled bioreactor cultivations of $R$. toruloides and the utilization efficiency of different substrates}

To compare the in silico predictions regarding the use of different substrates an initial set of cultivations was performed using glucose and glycerol as the sole carbon and energy source. The time profiles of the cultivations are shown in Figure 4. Typically, in the medium used, and with a fixed aeration rate of $0.5 \mathrm{vvm}$, four distinct culture phases were observed for both substrates: a growth phase under excess of both nutrient and oxygen (10 h or $12 \mathrm{~h}$ for growth on glucose and glycerol, respectively), a growth phase under oxygen-limited condition (last for about 10 and $20 \mathrm{~h}$ with glycerol and glucose respectively), and a phase with sufficient oxygen supply, presumably due to limitation by other nutrient(s) (which continued for almost $30 \mathrm{~h}$ or $40 \mathrm{~h}$ for glycerol and glucose respectively), and lastly a phase of oxygen

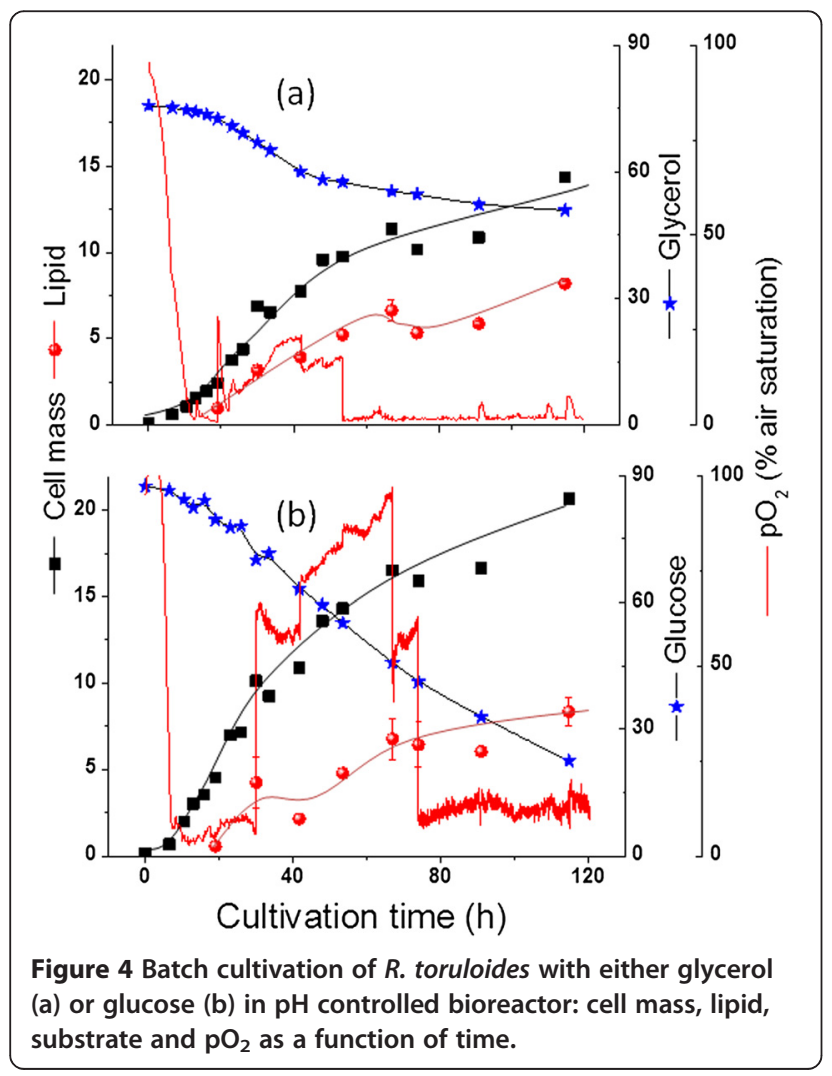

limitation again which continued till the end of the experiment.

The results shown that glucose supported a higher cell mass concentration with a maximum specific growth rate of $0.16 \mathrm{~h}^{-1}$ compared to $0.12 \mathrm{~h}^{-1}$ with glycerol as the sole carbon source. The lipid concentration on the other hand, was similar in both substrates ( $~ 8 \mathrm{~g} / \mathrm{L}$, Figure 4$)$. As predicted form the in silico analysis, most of the carbon was wasted as $\mathrm{CO}_{2}$ and cell mass if glucose was the sole carbon source and lipid accumulation never exceeded $45 \%$ (w/w) of cell mass. On the other hand lower cell mass but higher lipid content was observed with glycerol and a maximum lipid content of $57 \%$ (on cell mass basis) was achieved. Moreover, the lipid yield on glycerol consumed $\left(0.27 \mathrm{~g} . \mathrm{g}^{-1}\right)$ is more closer to the theoretical maximum value $\left(0.32{\mathrm{~g} . \mathrm{g}^{-1}}^{-1}\right.$ predicted than the lipid yield on glucose $\left(0.12 \mathrm{~g} . \mathrm{g}^{-1}\right.$ experimentally determined vs. $0.30 \mathrm{~g} . \mathrm{g}^{-1}$ of theoretical maximum). It should be emphasized that the maximum yield of TAG given in Table 1 was calculated for an optimal flux distribution where there is no cell mass production. Nevertheless, it is clear that an optimization of yield is especially needed for growth on glucose. Table 2 shows a comparison between the yields obtained by different $R$. toruloides strains on different substrates and conditions. The lipid yield on glucose could be improved by proper process control, especially by applying nitrogen or phosphorous limitations as demonstrated in literature. We found in this work that the lipid yield on glucose can be significantly improved by using blend with glycerol.

ATP requirements for growth, lipid production and maintenance are supplied mainly through the TCA cycle in the mitochondria. In fact, our in silico analysis was all done with a fixed P:O ratio of 1.2. The effects of oxidative phosphorylation (with varied $\mathrm{P}: \mathrm{O}$ ratio) on TAG yields using glucose as a substrate were analyzed using the proposed model. The change in P:O ratios (from 1.2 to 2) neither showed significant effect on the TAG yields (from 0.58 to $0.60 \mathrm{C}_{\mathrm{mol}} \cdot \mathrm{C}_{\mathrm{mol}}{ }^{-1}$ glucose), nor cell mass yield (0.67 to $0.71 \mathrm{C}_{\mathrm{mol}} \cdot \mathrm{C}_{\mathrm{mol}}{ }^{-1}$ glucose), respectively. Hence, it can be concluded that ATP generated by oxidative phosphorylation is not a major limiting factor for TAG synthesis. In agreement with the in silico analysis, controlling the oxygen concentration at $50 \%$ air saturation in the fermentation broth of $R$. toruloides grown either on glucose or glycerol throughout the cultivation process did not have any significant effect on the yield (Table 2). Similar to the cultivations without $\mathrm{pO}_{2}$ control (e.g. O2 limitation in phases 2 and 4, see Figure 4), the maximal growth rate with glucose was $0.23 \mathrm{~h}^{-1}$ compared to $0.13 \mathrm{~h}^{-1}$ with glycerol in $\mathrm{pO}_{2}$ controlled cultivations. The maximum lipid content on glycerol was $61 \%$ vs. $57 \%$ in $\mathrm{pO}_{2}$ uncontrolled culture, whereas it was $48 \%$ on glucose vs. $41 \%$ in $\mathrm{pO}_{2}$ uncontrolled culture. The lipid productivity increased significantly in the $\mathrm{pO}_{2}$ 
Table 2 Experimental lipid yields and productivity of different $\boldsymbol{R}$. toruloides strains grown on different substrates

\begin{tabular}{|c|c|c|c|c|c|}
\hline Carbon source & $\begin{array}{l}\text { Lipid productivity } \\
\text { (g/L/h) }\end{array}$ & Condition & $\begin{array}{l}\text { Lipid yield }{ }^{*} \\
\text { (g/g substrate) }\end{array}$ & $\begin{array}{l}\text { Lipid yield } \\
\text { (C-mol.C-mol }^{-1} \\
\text { substrate) }\end{array}$ & Reference \\
\hline glucose & 0.15 & Batch- N limiting, pO2 controlled & 0.15 & 0.29 & \multirow[t]{10}{*}{ This work } \\
\hline glycerol & 0.06 & Batch- N limiting, pO2 controlled & 0.24 & 0.47 & \\
\hline glucose + glycerol & 0.12 & Batch- N limiting, pO2 controlled & 0.17 & 0.33 & \\
\hline glucose & 0.07 & Batch- N limiting, pO2 un-controlled & 0.12 & 0.23 & \\
\hline glycerol & 0.06 & Batch- N limiting, pO2 un-controlled & 0.27 & 0.53 & \\
\hline glucose + glycerol & 0.07 & Batch- N limiting, pO2 un-controlled & 0.2 & 0.38 & \\
\hline Glucose + glycerol + xylose & \multirow[t]{2}{*}{0.08} & \multirow[t]{2}{*}{ Batch- $\mathrm{N}$ limiting, pO2 controlled } & \multirow[t]{2}{*}{0.22} & \multirow[t]{2}{*}{0.42} & \\
\hline$(10: 20: 30)$ & & & & & \\
\hline Biomass hydrolysate + glycerol & \multirow[t]{2}{*}{0.09} & \multirow[t]{2}{*}{ Batch- $\mathrm{N}$ limiting, pO2 controlled } & \multirow[t]{2}{*}{0.17} & \multirow[t]{2}{*}{-} & \\
\hline (glucose- xylose - glycerol =10:20:30) & & & & & \\
\hline glucose & - & Batch, phosphorous limiting & 0.21 & - & [17] \\
\hline glucose & - & Continuous- nitrogen limiting & 0.19 & - & {$[38]$} \\
\hline
\end{tabular}

TAG molecule with 51 carbons and a molecular weight of $801.27 \mathrm{~g} / \mathrm{mol}$ are used to calculate the yields in C-mol/C-mol.

*lipid yield was calculated from the slope between lipid concentration against consumed sugar(s).

controlled experiments, mainly because of the increased growth rates.

Blends of sugars and glycerol were also tested for the production of lipid by $R$. toruloides. Interestingly, this strain showed a catabolite repression in the presence of glucose as depicted in Figure 5a. Catabolite repression was not observed before in certain oleaginous yeasts. Strains of $Y$. lipolytica and $R$. glutinis were shown previously to metabolize both carbon sources simultaneously $[23,25,26]$.

As shown in Figure 5a, a clear diauxic growth was observed in the mixed substrate cultivations: glycerol was consumed only after glucose consumption in this case. Despite of the observed catabolite repression, the addition of glycerol enhanced the lipid yield on glucose significantly (Figure 5b), confirming the predictions from the in silico analysis. In fact, the mixed substrate cultivation has the dual advantages of efficient cell mass production (normally maximized with glucose) with higher lipid content (obtained normally with glycerol). The lipid yields on the substrate consumed and the specific lipid productivities on cell mass are higher when glucose and glycerol are used in mixture rather than only glucose (Table 2).

In the above blend substrate fermentation experiments, glucose was used at a concentration of $10 \%$ of the added carbon source. The effect of different combinations of the substrates on the lipid yield was further investigated in flask experiments. As compared to mono-substrate fermentation with glucose, a $26 \%$ increase of lipid yield was observed if glycerol is added (Figure 6). Similarly to the above results from controlled bioreactors, glucose as mono-substrate

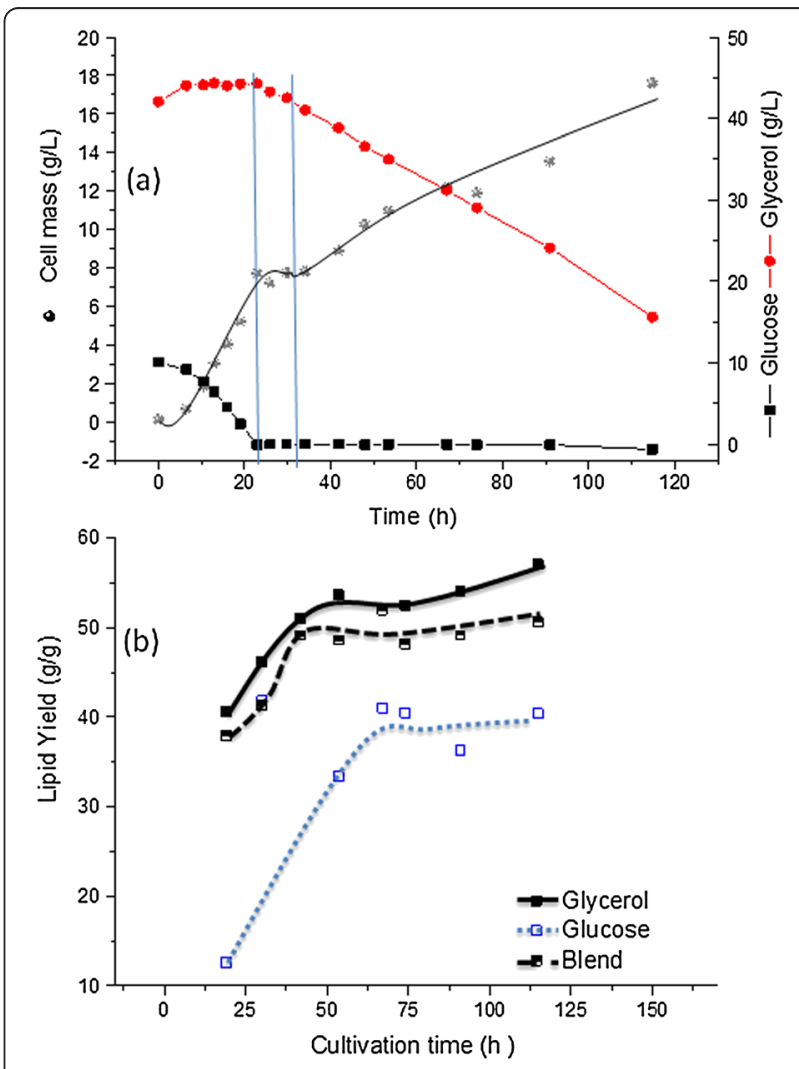

Figure $5 \mathrm{pO}_{2}$ uncontrolled cultivation of $R$. toruloides and the diauxy growth behavior on a mixture of glucose and glycerol $(10: 90 \mathrm{~g} / \mathrm{g}(\mathrm{a}))$, and a comparison of the different substrate and blend on the lipid yield on produced cell mass (b). 


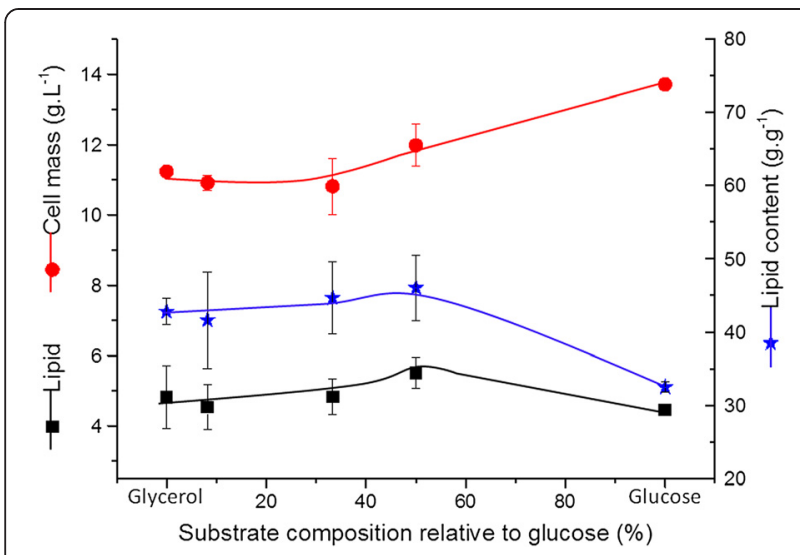

Figure 6 Cell mass and lipid production by $R$. toruloides grown on mono-substrate and on different variation of dual substrate fermentation.

supported the highest cell mass production (13.7 g.. $\left.\mathrm{L}^{-1}\right)$ with the lowest lipid content (32\%). Similarly, with a closely related yeast strain $R$. glutinis, Easterling [23] reported that blend substrate fermentation produced more cell mass than when a single carbon source was provided. They also reported that the lipid content was minimum with glucose and significantly higher with either glycerol or blend of both. However, they did not report catabolite repression for R. glutinis. However, it should be stressed that, on mixtures of carbohydrates and with the sequential utilization of sugars, the sugar is being consumed with more nitrogen available (and hence more cell mass formation), followed by glycerol consumption during a period of nitrogen restricted growth which will have a positive effect on the lipid yield. Still, the mechanism of the positive role of glycerol for the lipid synthesis of cells grown in blends deserves more detailed studies. Glycerol can be utilized as a source of carbon and energy. But under certain conditions (e.g. in pO2-controlled culture with glucose as the sole carbon source), $R$. toruloides can produce glycerol extracellularly up to $5 \mathrm{~g} / \mathrm{L}$ (data not shown). The two most important functions of glycerol synthesis in yeast are related to redox balancing and the hyperosmotic stress response [27]. In Saccharomyces cerevisiae, production of glycerol occurs to maintain the redox balance. Interestingly, glycerol production is found to be higher in a minimal medium than in a complex medium [28]. It was concluded that in a minimal medium the de novo synthesis of amino acids from glucose and ammonia leads to an excess of NADH which is reoxidized by glycerol formation. In osmotolerant yeasts, glycerol plays a crucial role in their survival [29]. Recently, Petelenz-kurdziel et al. [30] reported that S. cerevisiae adapts to hyperosmotic stress by activating the HOG signaling cascade, which controls glycerol accumulation, production and import. Moreover, it was also shown that glycerol transport depends on the strain and the growth conditions. Glycerol production has been never reported in $R$. toruloides, and hence transcriptomic analysis is being further performed in our laboratory.

The effects of different growth substrates and culture conditions on the fatty acid composition were also studied (Figure 7). In oxygen controlled conditions at $\mathrm{pO}_{2}$ of $50 \%$ of air saturation, and on all the substrates and blends used, palmitic, oleic and linoleic acid constitute more than $90 \%$ of the total fatty acids. In oxygen uncontrolled cultivation (constant aeration and agitation, $\mathrm{pO}_{2}$ not controlled), about $65 \%$ of the fatty acid measured is unsaturated (oleic acid and linoleic acid) if glycerol or blend are used, compared to $57 \%$ of saturated fatty acids (lauric acid, stearic acid and palmitic acid) if glucose is the sole carbon source. Oxygen limitation has significantly influenced the fraction of saturated and unsaturated fatty acid composition in the lipid produced by R.toruloides especially on glycerol or mixed substrates. Oxygen controlled conditions derived fatty acid compositions that are suitable for biodiesel conversion [9] whereas oxygen un-controlled conditions produced significant amounts of saturated fatty acids which are close to be substitutes of certain important products like cocoa butter.

$R$. toruloides is a known lipogenic yeast and the results presented here confirm its ability to accumulate over

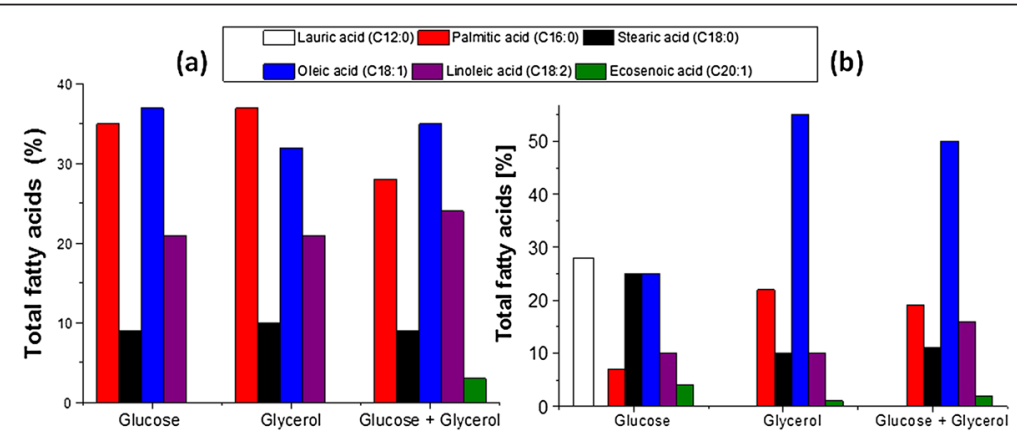

Figure 7 Fatty acid composition of lipid produced by R.toruloides grown on glucose or glycerol and on mixture. a. Fatty acid composition from a pO2 controlled cultivation, $\mathbf{b}$. fatty acid composition on a pO2 un-controlled cultivation. 
$60 \%$ of its cell mass as lipid. However, lipid accumulation was strongly affected by the nature of the carbon source provided. The recently sequenced and annotated genome of $R$. toruloides, its proteomic analysis and the available protein annotation have opened doors to understand the underlying mechanism of this organism for lipid production [31-33]. In this work, elementary modes were used to decompose the complex metabolic network into its basic functioning units and to identify the potentials of triacyglyceride production on different substrates in $R$. toruloides. Maximum theoretical TAG yields on hexoses (glucose), pentose (xylose and arabionse), glycerol and on mixture of hexoses \& glycerol, pentose \& glycerol have been estimated (Table 1) and were in general agreement with previously reported theoretical maximum yields $[34,35]$. As shown from the computed solution span in Additional file 1: Figure S1, the production is still far from the theoretical optimum and the distance between the actual production state on glucose or glycerol and the theoretical optimum suggests an enormous potential for future optimization. In silico optimization, identification of limiting steps and finally suggestions for future genetic modification are discussed below.

One of the key processes for fatty acid biosynthesis is the provision of reducing power (e.g. NADPH) to reduce the acetyl group and channel it into the acyl chain of fatty acid. In Y. lipolytica, the PP pathway is assumed to be the sole supplier of NADPH for lipid synthesis, since it does not possess a cytosolic malic enzyme [36] and the deletion of mitochondrial malic enzyme did not show any effect on lipid production [37]. In R. toruloides, an alternative NADPH supplying reaction would be the cytosolic NADP-dependent isocitrate dehydrogenase (ICDH) which is inactive in the predicted optimal flux distribution of glucose (Figure 1). A single gene deletion study of our model was performed to remove the cytosolic malic enzyme from the reactions. A reduction in maximum theoretical yield of TAG was observed (from 0.30 g.g $\mathrm{g}^{-1}$ to $\left.0.27 \mathrm{~g} . \mathrm{g}^{-1}\right)$ on glucose and glycerol $\left(0.32 \mathrm{~g} . \mathrm{g}^{-1}\right.$ to $\left.0.27 \mathrm{~g} . \mathrm{g}^{-1}\right)$. However, the theoretical maximum yield retained to $0.30 \mathrm{~g} . \mathrm{g}^{-1}$ on glucose and $0.32 \mathrm{~g} . \mathrm{g}^{-1}$ on glycerol if a NADP-dependent acetaldehyde dehydrogenase (ALD6) is incorporated. This enzyme constitutively catalyzes the conversion of acetaldehyde to acetate, generating an NADPH in the cytoplasm. Another alternate route for supplying NADPH as proposed by Ratledge [34] can be a mitochondrial NAD-dependent ICDH acting in the reverse direction. EFM analysis and optimal flux distribution were done (Additional file 1: Figure S5) and the maximum theoretical yield of TAG on glucose is retained to $0.30 \mathrm{~g} \cdot \mathrm{g}^{-1}$ glucose. A third possible target could be a heterologous NADPH generating enzyme or an endogenous engineered NADPH generating enzyme, both in the glycolytic pathway [38] which could be a potential source for NADPH. By changing GAPDH, which is a NAD-dependent glyceraldehyde dehydrogenase in the glycolytic pathway, to an NADP-dependent enzyme can increase the theoretical TAG yields by $7 \%$ on glucose and $9 \%$ on glycerol.

Transhydrogenases for converting NADH to NADPH could be another alternative source for enhancing the availability of NADPH. Nevertheless, oleaginous yeasts or fungi have never been reported to harbor a transhydrogenase. Bacterial cells on the other hand, possess transhydrogenase activity. Recent genome sequencing and annotation has predicted a NADP-transhydrogenase like protein (RTG_ 03342) in Rhodotorula glutinis ATCC 204091 [39] with a UniProtKB id GOT2B1. This protein is $84 \%$ identical to an uncharacterized protein of $R$. toruloides (RHTO_06438). A theoretical analysis of the metabolic implications of a transhydrogenase reaction was investigated using the $R$. toruloides model. About 10\% higher theoretical maximum yields were calculated on all the substrates when a transhydrogenase activity was introduced (Table 3). The optimal

Table 3 Elementary modes (EMs) and theoretical TAG yields with heterologous genes/reactions

\begin{tabular}{|c|c|c|c|c|}
\hline Carbon source & EMs & Characteristics & $\begin{array}{l}\text { Maximum yield of TAG } \\
{\left[\mathrm{C}-\mathrm{mol} . \mathrm{C}-\mathrm{mol}^{-1} \text { substrate] }\right.}\end{array}$ & $\begin{array}{l}\text { Maximum yield of TAG } \\
{\left[\mathrm{g} \cdot \mathrm{g}^{-1} \text { substrate] }\right.}\end{array}$ \\
\hline Glucose & 30,132 & Transhydrogenase & 0.64 & 0.33 \\
\hline Glycerol & 57,172 & Transhydrogenase & 0.68 & 0.35 \\
\hline Xylose & 21,323 & Transhydrogenase & 0.64 & 0.33 \\
\hline Arabinose & 27,264 & Transhydrogenase & 0.64 & 0.33 \\
\hline Glucose & 26,878 & Cytosolic NADP-dependent GAPDH & 0.62 & 0.32 \\
\hline Glycerol & 42,578 & Cytosolic NADP-dependent GAPDH & 0.67 & 0.35 \\
\hline Glucose & 6858 & Without cytosolic Malic enzyme & 0.51 & 0.27 \\
\hline Glucose & 12,487 & With NADP-dependent ALD6 & 0.58 & 0.30 \\
\hline Glycerol & 23,658 & With NADP-dependent ALD6 & 0.63 & .032 \\
\hline Glucose & 15,331 & Cytosolic NADH source & 0.68 & 0.36 \\
\hline Glycerol & 35,804 & Cytosolic NADH source & 0.68 & 0.35 \\
\hline
\end{tabular}


modes with the highest lipid yield do not include PP pathway flux but a high flux through the glycolysis is found which generates the cytosolic NADH for conversion to NADPH through the transhydrogenase reaction. On all sugars about $10 \%$ of the flux is also observed for the cytosolic NADP-dependent ICDH providing $\mathrm{NADPH}$ other than the transhydrogenase for TAG synthesis. On glycerol the sole NADPH supplier is found to be the transhydrogenase reaction. With the aid of already established genetic engineering techniques [40] for R.toruloides, the predicted modifications should facilitate the development of superior lipid producing strains.

\section{Conclusions}

The present work describes the successful application of reconstruction of metabolic network and it's in silico analysis for determining and understanding the potential and metabolic pathways for lipid production in $R$. toruloides grown on different substrates, especially for substrate blends. Cultivations of $R$. toruloides were carried out with different substrates and in a well-controlled bioreactor system. The experimental results are in general agreement with model predictions in terms of lipid yield.

The constructed metabolic model can be used to guide further optimization of lipid synthesis in this strain. The maximal lipid yield experimentally achieved is still significantly below the theoretical maximum, especially for growth on glucose $(0.29$ vs. $0.58 \mathrm{C}-\mathrm{Mol} / \mathrm{C}-\mathrm{Mol})$ and its blend with glycerol. Several strategies to increase the lipid yield are identified and discussed with the help of the metabolic model.

\section{Methods}

\section{Microorganism and media}

$R$. toruloides DSMZ 4444 was used for the current study. The strain was maintained at $-80^{\circ} \mathrm{C}$ on potato dextrose medium with $20 \%$ glycerol (v/v). The medium for seed cultures and main cultures was a nitrogen limited medium which is similar to that reported previously [26]. Carbon sources (glucose, glycerol, xylose, arabinose or biomass hydrolysates) were autoclaved separately and added together with sterile $\mathrm{FeCL}_{3}$ and $\mathrm{CaCl}_{2}$ solutions and inoculated immediately. Spruce BH (Borregard, Norway) was also used in co-substrate fermentation with glycerol. An enzymatic hydrolysis of spruce was done without buffer. The hydrolysate was then heated at $80^{\circ} \mathrm{C}$ for $15-20 \mathrm{~min}$ to inactivate the enzymes. The supernatant was removed and filtered with a centrifuge with filter bag. The sample was then concentrated by vacuum evaporation at $60^{\circ} \mathrm{C}$. The concentrated hydrolysate contained $550 \mathrm{~g} / \mathrm{L}$ glucose and $35 \mathrm{~g} / \mathrm{L}$ xylose.

\section{Cultivations}

Seed cultures and cultivations in flasks were performed in baffled shake flasks incubated at $30^{\circ} \mathrm{C}$ and $180 \mathrm{rpm}$ for $24 \mathrm{~h}$. Batch cultivations were performed in a $1.5 \mathrm{~L}$ well-equipped parallel bioreactor system (DASGIP parallel bioreactor system, Jülich, Germany) with $1 \mathrm{~L}$ initial working volume. Cultivations were started by inoculating $30 \mathrm{~mL}$ (3\%) from the seed cultures grown for $24 \mathrm{~h}$. $\mathrm{pH}$ was maintained at 6.0 using $5 \mathrm{~N} \mathrm{NaOH}$ and $2 \mathrm{M}$ HCL. Dissolved oxygen was maintained at $50 \%$ air saturation. Carbon dioxide evolution rates and oxygen uptake rates were automatically evaluated by the online DASGIP off gas analyzing system equipped with sensors from Bluesens.

\section{Analytical methods}

Cell growth was recorded as optical density at $600 \mathrm{~nm}$. Cell mass was harvested during the cultivations after centrifugation $\left(5000 \mathrm{rpm}, 10 \mathrm{~min}\right.$ at $\left.4^{\circ} \mathrm{C}\right)$. Cell dry weight was determined gravimetrically after drying the harvested cells in an oven at $80^{\circ} \mathrm{C}$ to a constant weight. Extraction of lipids was performed with a modified method described previously using Folsch solution (Chloroform: Methanol $=2: 1$ vol.vol $^{-1}$, [41]. Non-lipid cell mass was calculated after subtraction of intracellular lipids from the total cell mass. Quantification of glucose, glycerol and organic acids was carried out using high-performance liquid chromatography (HPLC; Kontron Instruments, United Kingdom) with separation on an Aminex HPX-87H column at $60^{\circ} \mathrm{C}$ with $0.005 \mathrm{M} \mathrm{H}_{2} \mathrm{SO}_{4}$ and detection via refractive index or by UV absorption at $210 \mathrm{~nm}$. Ammonia concentration in the supernatant was determined by photometric measurements using a kit from Macherey Nagel, Germany. GC analysis of the fatty acid methyl esters was performed as reported [42] with a Varian 3900 gas chromatograph equipped with a flame ionization detector (FID) and a TR-FAME column (Thermo Scientific, Germany, 50 m X 0.22 mm X $0.25 \mu \mathrm{m}$ ).

\section{Metabolic network and elementary mode analysis}

A metabolic network with the most important reactions of $R$. toruloides was reconstructed and is depicted in Figure 8. A complete list of the considered reactions is represented in the Additional file 1: Table S1. The metabolic network model was built manually by considering the available genome sequence and proteomic evidences [33] from UniProtKB. Briefly, the model includes all relevant pathways of central carbon metabolism comprising of glucose, glycerol, xylose and arabinose uptakes. Triacylglycerol (TAG) synthesis is assumed to be formed from glycerol and $C_{16}$ equivalent fatty acids (i.e. palmitic acid) as shown in the reaction network (Additional file 1: Table S1). Assuming that the TAG (C57, M.W $=885.4 \mathrm{~g} / \mathrm{mol}$ ) is formed from glycerol and $\mathrm{C}_{18}$ oleic acid, it did not show any 


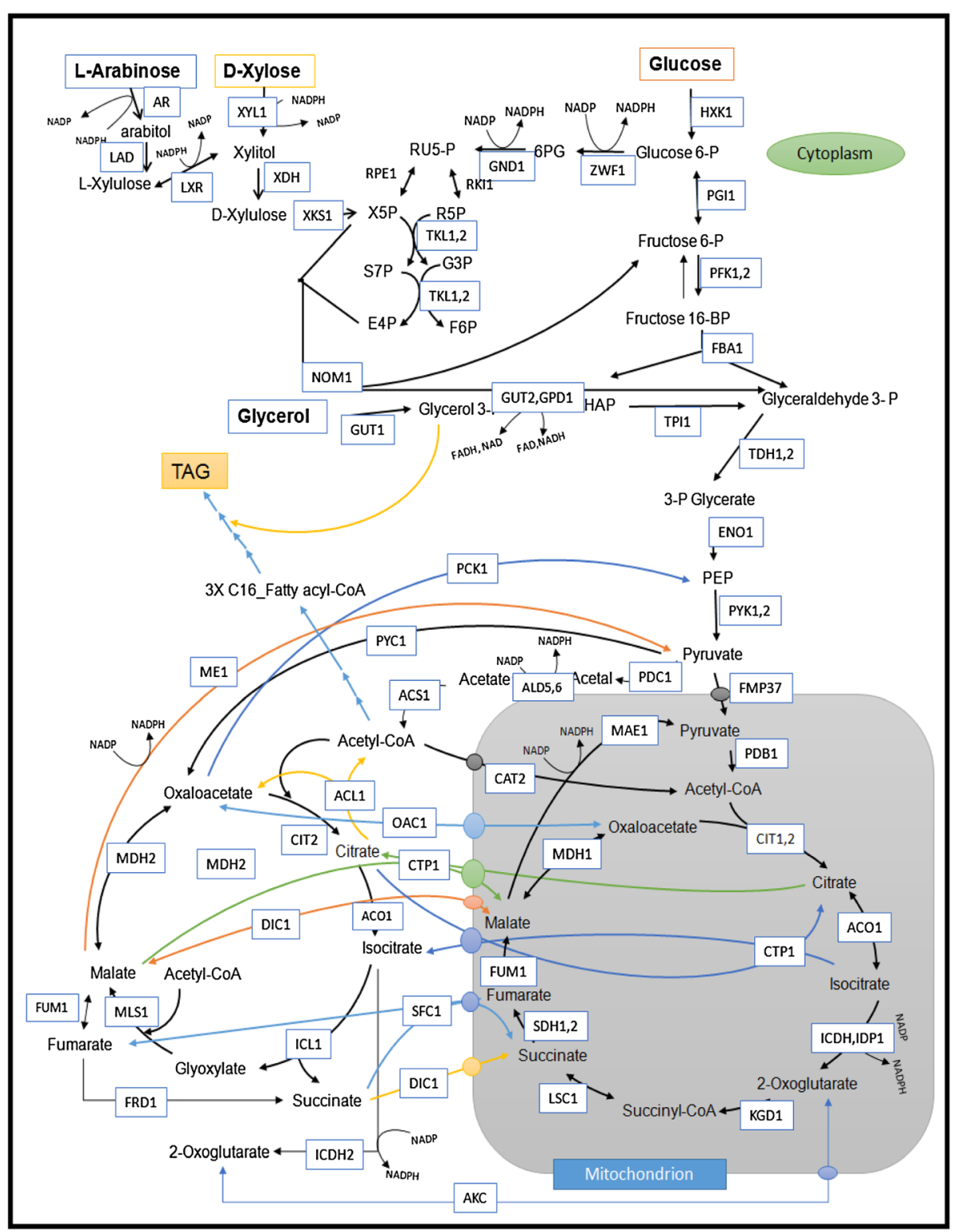

Figure 8 Metabolic model of Triacylglycerol producing Rhodosporidium toruloides.

difference in the yields from that were observed based on $\mathrm{C}_{16}$ palmitic acid assumption. So, $\mathrm{C}_{16}$ palmitic acid was used for analysis for all the considered in silico predictions. Cellular compartments mitochondrion and cytosol were considered together with respective transport reactions. For elementary mode analysis (EFM), the influx of substrates (glucose, glycerol, xylose, arabinose, oxygen, nitrogen and sulphur) and efflux of products (triacylglyceride, cell mass, carbon dioxide and ATP for maintenance) are considered. The $\mathrm{P} / \mathrm{O}$ ratio is taken as 1.2 and the cell mass equation is taken from $S$. cerevisiae's data [43]. EFMs were calculated using the software tool CellNetAnalyzer [44] in MATLAB 2013a. The EFMs were sorted and the theoretical yields on different substrates were calculated using Microsoft Excel. EFMs correspond to minimal functional pathways of a metabolic network and are useful to study various functional network properties $[45,46]$.

The metabolic network with D-glucose as a substrate comprises of 69 reactions, of which 27 are reversible with 61 internal metabolites and 8 external metabolites. With glycerol as a sole carbon source, the network is comprised of 71 reactions ( 28 reversible) of which 61 are internal and 8 external metabolites. The network with $\mathrm{D}$-xylose and L-arabinose comprises 71 and 73 reactions respectively. Theoretical maximum yields were calculated based on the obtained fluxes. All fluxes are given in $\mathrm{mmol} \cdot \mathrm{g}^{-1} \cdot \mathrm{h}^{-1}$ normalized to the substrate uptake rate. 


\section{Additional file}

Additional fie 1: Table S1. In silico pathway analysis and lipid production of Rhodosporidium toruloides on mixed substrates. Figure S1. The biomass yield [c-mol.c-mol ${ }^{-1}$ ] on corresponding substrates is plotted against the TAG yield on the substrate which is in c-mol.c-mol ${ }^{-1}$. Theoretical maximum yields on each substrate with and without biomass formation are represented. Figure S2. Optimal flux distribution on xylose. All values are relative molar fluxes $\left(\mathrm{mmol} . \mathrm{g}^{-1} \mathrm{~h}^{-1}\right)$ normalized to the xylose uptake rate. Figure S3. Optimal flux distribution on arabinose. All values are relative molar fluxes $\left(\mathrm{mmol} \cdot \mathrm{g}^{-1} \mathrm{~h}^{-1}\right)$ normalized to the arabinose uptake rate. Figure S4. Optimal flux distribution on xylose and glycerol. All values are relative molar fluxes (mmol. $\mathrm{g}^{-1} \mathrm{~h}^{-1}$ ) normalized to the xylose uptake rate. Figure S5. Optimal flux distribution on glucose with ICDH acting in reverse direction and without cytosolic malic enzyme. All values are relative molar fluxes (mmol. $\mathrm{g}^{-1} \mathrm{~h}^{-1}$ ) normalized to the xylose uptake rate.

\section{Competing interests}

The authors declare that they have no competing interests.

\section{Authors' contributions}

RRB and WS contributed equally in the design and interpretation of the experiments and results. RRB constructed the metabolic pathway, did the in silico analysis and interpretations. RRB and WS designed the experiments and performed the experiments with GM. RRB and WS wrote and drafted the manuscript. APZ supervised the research. All authors read and approved the final manuscript.

\section{Acknowledgements}

The financial support of this investigation from the BMBF under the project 'Bio4Oil project number: 03SF0467' is gratefully acknowledged. We thank the project partners Prof. Geoge Aggelis from the University of Patra und Prof. Seraphim Papanikoloau from Agricultural University of Athens for helpful discussion. We also thank Dr. Wei Wang for helping to set-up the GC method for lipid analysis.

\section{Received: 24 December 2014 Accepted: 24 February 2015} Published online: 18 March 2015

\section{References}

1. Yang F, Hanna MA, Sun R. Value-added uses for crude glycerol-a byproduct of biodiesel production. Biotechnol Biofuels. 2012;5:13.

2. Xu J, Zhao X, Wang W, Du W, Liu D. Microbial conversion of biodiese byproduct glycerol to triacylglycerols by oleaginous yeast Rhodosporidium toruloides and the individual effect of some impurities on lipid production. Biochem Eng J. 2012;65:30-6.

3. Kiran EU, Trzcinski A, Webb C. Microbial oil produced from biodiesel by-products could enhance overall production. Bioresour Technol. 2013;129:650-4.

4. Chatzifragkou A, Makri A, Belka A, Bellou S, Mavrou M, Mastoridou M, et al. Biotechnological conversions of biodiesel derived waste glycerol by yeast and fungal species. Energy. 2011;36:1097-108.

5. Dietz D, Zeng AP. Efficient production of 1,3-propanediol from fermentation of crude glycerol with mixed cultures in a simple medium. Bioprocess Biosyst Eng. 2014;37:225-33.

6. Kaeding T, DaLuz J, Kube J, Zeng AP. Integrated study of fermentation and downstream processing in a miniplant significantly improved the microbial 1,3-propanediol production from raw glycerol. Bioprocess Biosyst Eng. 2014, Nov 4. [Epub ahead of print]

7. Moore SJ, Mayer MJ, Biedendieck R, Deery E, Warren MJ. Towards a cell factory for vitamin B12 production in Bacillus megaterium: bypassing of the cobalamin riboswitch control elements. N Biotechnol. 2014;31:553-61.

8. Papanikolaou S, Beopoulos A, Koletti A, Thevenieau F, Koutinas AA, Nicaud $\mathrm{JM}$, et al. Importance of the methyl-citrate cycle on glycerol metabolism in the yeast Yarrowia lipolytica. J Biotechnol. 2013;168:303-14.

9. Minkevich IG, Dedyukhina EG, Chistyakova TI. The effect of lipid content on the elemental composition and energy capacity of yeast biomass. Appl Microbiol Biotechnol. 2010;88:799-806.

10. Papanikolaou S, Aggelis G. Lipid of oleaginous yeast. Part I: biochemistry of single cell oil production. Eur J Lipid Sci Technol. 2011;113:1031-51.
11. Liu B, Zhao ZK. Biodiesel production by direct methanolysis of oleaginous microbial biomass. J Cem Technol Biotechnol. 2007:82:775-80.

12. Papanikolaou S, Muniglia L, Chevalot I, Aggelis G, Marc I. Accumulation of a cocoa-butter-like lipid by Yarrowia lipolytica cultivated on agro-industrial residues. Curr Microbiol. 2003;46:124-30

13. Zhao X, Kong X, Hua Y, Feng B, Zhao ZK. Medium optimization for lipid production through co-fermentation of glucose and xylose by the oleaginous yeast Lipomyces starkeyi. Eur J Lipid Sci Technol. 2008;110:405-12.

14. Gong Z, Wang Q, Shen H, Wang L, Xie H, Zhao ZK. Conversion of biomassderived oligosaccharides into lipids. Biotechnol Biofuels. 2014;7:13.

15. Koutinas AA, Vlysidis A, Pleissner D, Kopsahelis N, Kookos IK, Papanikolaou S, et al. Valorization of industrial waste and by-product streams via fermentation for the production of chemicals and biopolymers. Chem Soc Rev. 2014;43:2587-627.

16. Muller EE, Sheik AR, Wilmes P. Lipid-based biofuel production from wastewater. Curr Opin Biotechnol. 2014;30C:9-16.

17. Wiebe MG, Koivuranta K, Penttila M, Ruohonen L. Lipid production in batch and fed-batch cultures of Rhodosporidium toruloides from 5 and 6 carbon carbohydrates. BMC Biotechnol. 2012;12:26.

18. Zhou W, Wang W, Li Y, Zhang Y. Lipid production by Rhodosporidium toruloides $Y 2$ in bioethanol wastewater and evaluation of biomass energetic yield. Bioresour Technol. 2013;127:435-40.

19. Ageitos JM, Vallejo JA, Veiga-Crespo P, Villa TG. Oily yeasts as oleaginous cell factories. Appl Microbiol Biotechnol. 2011;90:1219-27.

20. Jin G, Zhang Y, Shen H, Yang X, Xie H, Zhao ZK. Fatty acid ethyl esters production in aqueous phase by the oleaginous yeast Rhodosporidium toruloides. Bioresour Technol. 2013;150:266-70.

21. Wu S, Hu C, Jin G, Zhao X, Zhao ZK. Phosphate-limitation mediated lipid production by Rhodosporidium toruloides. Bioresour Technol. 2010;101:6124-9.

22. Yang X, Jin G, Gong Z, Shen H, Bai F, Zhao ZK. Recycling biodiesel-derived glycerol by the oleaginous yeast Rhodosporidium toruloides $Y 4$ through the two-stage lipid production process. Biochem Eng J. 2014;91:86-91.

23. Easterling ER, French WT, Hernandez R, Licha M. The effect of glycerol as a sole and secondary substrate on the growth and fatty acid composition of Rhodotorula glutinis. Bioresour Technol. 2009;100:356-61.

24. Fonseca C, Romao R, Sousa HR, Hägerdal BH, Martins IS. L-Arabinose transport and catabolism in yeast. FEBS. 2007:274:3589-600.

25. Workman M, Holt P, Thykaer J. Comparing cellular performance of Yarrowia lipolytica during growth on glucose and glycerol in submerged cultivations. AMB Express. 2013;3:58.

26. Papanikolaou S, Muniglia L, Chevalot I, Aggelis G, Marc I. Yarrowia lipolytica as a potential producer of citric acid from raw glycerol. J Appl Microbiol. 2002;92:737-44.

27. Jain VK, Divol B, Prior BA, Bauer FF. Elimination of glycerol and replacement with alternative products in ethanol fermentation by Saccharomyces cerevisiae. J Ind Microbiol Biotechnol. 2011:38:1427-35.

28. Albers E, Larsson C, Liden G, Niklasson C, Gustafsson L. Influence of the nitrogen source on Saccharomyces cerevisiae anaerobic growth and product formation. Appl Environ Microbiol. 1996;62:3187-95.

29. Hohmann S. Osmotic stress signaling and osmoadaptation in yeasts. Microbiol Mol Biol Rev. 2002;66:300-72.

30. Petelenz-Kurdziel E, Kuehn C, Nordlander B, Klein D, Hong KK, Jacobson T, et al. Quantitative analysis of glycerol accumulation, glycolysis and growth under hyper osmotic stress. PLoS Comput Biol. 2013;9:e1003084.

31. Kumar S, Kusheaha H, Bachhawat AK, Raghava GP, Ganesan K. Genome sequence of the oleaginous red yeast Rhodosporidium toruloides MTCC 457 Eukaryot Cell. 2012;11:1083-4.

32. Liu H, Zhao X, Wang F, Li Y, Jiang $X$, Ye M, et al. Comparative proteomic analysis of Rhodosporidium toruloides during lipid accumulation. Yeast. 2009;26:553-66.

33. Zhu Z, Zhang S, Liu H, Shen $H$, Lin $X$, Yang F, et al. A multi-omic map of the lipid-producing yeast Rhodosporidium toruloides. Nat Commun. 2012;3:1112.

34. Ratledge $\mathrm{C}$. The role of malic enzyme as the provider of NADPH in oleaginous microorganisms: a reappraisal and unsolved problems. Biotechnol Lett. 2014;36:1557-68.

35. Ratledge C, Wynn JP. The biochemistry and molecular biology of lipid accumulation in oleaginous microorganisms. Adv Appl Microbiol. 2002;51:1-51.

36. Dujon B, Sherman D, Fischer G, Durrens P, Casaregola S, Lafontaine I, et al. Genome evolution in yeasts. Nature. 2004;430:35-44. 
37. Zhang $H$, Zhang L, Chen $H$, Chen $Y Q$, Raltedge $C$, Song $Y$, et al. Regulatory properties of malic enzyme in the oleaginous yeast, Yarrowia lipolytica, and its non-involvement in lipid accumulation. Biotechnol Lett. 2013;35:2091-8

38. Bommareddy RR, Chen Z, Rappert S, Zeng AP. A de novo NADPH generation pathway for improving lysine production of Corynebacterium glutamicum by rational design of the coenzyme specificity of glyceraldehyde 3-phosphate dehydrogenase. Metab Eng. 2014;25:30-7.

39. Paul D, Magbanua Z, Arick M, French T, Bridges SM, Burgess SC, et al. Genome sequence of the Oleaginous Yeast Rhodotorula glutinis ATCC 204091. Genome Announc. 2014;2:e00046-14.

40. Lin $X$, Wang Y, Zhang S, Zhu Z, Zhou YJ, Yang F, et al. Functional integration of multiple genes into the genome of the oleaginous yeast Rhodosporidium toruloides. FEMS Yeast Res. 2014;14:547-55.

41. Papanikolaou S, Galiotou-Panayotou M, Chevalot I, Komaitis M, Marc I, Aggelis $\mathrm{G}$. Influence of glucose and saturated free-fatty acid mixtures on citric acid and lipid production by Yarrowia lipolytica. Curr Microbiol. 2006;52:134-42.

42. Beopoulos A, Mrozova Z, Thevenieau F, Le Dall MT, Hapala I, Papanikolaou $\mathrm{S}$, et al. Control of lipid accumulation in the yeast Yarrowia lipolytica. Appl Environ Microbiol. 2008;74:7779-89.

43. Gruchattka E, Hadicke O, Klamt S, Schutz V, Kayser O. In silico profiling of Escherichia coli and Saccharomyces cerevisiae as terpenoid factories. Microb Cell Fact. 2013;12:84.

44. Klamt S, Saez-Rodriguez J, Gilles ED. Structural and functional analysis of cellular networks with Cell NetAnalyzer. BMC Syst Biol. 2007;1:2.

45. Schuster S, Fell DA, Dandekar T. A general definition of metabolic pathways useful for systematic organization and analysis of complex metabolic networks. Nat Biotechnol. 2000;18:326-32.

46. Trinh CT, Wlaschin A, Srienc F. Elementary mode analysis: a useful metabolic pathway analysis tool for characterizing cellular metabolism. Appl Microbiol Biotechnol. 2009;81:813-26.

\section{Submit your next manuscript to BioMed Central and take full advantage of:}

- Convenient online submission

- Thorough peer review

- No space constraints or color figure charges

- Immediate publication on acceptance

- Inclusion in PubMed, CAS, Scopus and Google Scholar

- Research which is freely available for redistribution 